\title{
Primary Cutaneous Diffuse Large B-Cell Lymphoma (Leg Type) Presenting as Necrotising Fasciitis
}

\author{
Niall M. McInerney ${ }^{*}$, Kieran T. Power, Alan J. Hussey \\ Department of Plastic and Reconstructive Surgery, Galway University Hopsital, Galway, Ireland. \\ Email: *nmac@ymail.com
}

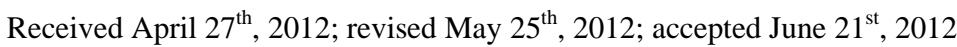

\begin{abstract}
Necrotising fasciitis is a rare rapidly progressive, life threatening, soft tissue infection which spreads along fascial planes. We present a patient who was diagnosed with a primary cutaneous Diffuse Large B-Cell Lymphoma (leg type) following initial presentation as probable necrotising fasciitis. Presentation was of a painful swollen leg, septic shock and MRI findings consistent with the clinical signs. Diagnosis of necrotising fasciitis remains challenging and it is often missed in the early stages. Early fascial biopsy and histopathological analysis is useful in cases where the diagnosis is unclear. We feel that this case highlights these important issues and will benefit others in their management of similar cases in the future.
\end{abstract}

Keywords: Cutaneous; Lymphoma; Necrotising; Fasciitis

\section{Introduction}

Necrotising fasciitis is a rare rapidly progressive, life threatening, soft tissue infection which spreads along fascial planes [1]. If left untreated it is invariably fatal. A high index of clinical suspicion is therefore warranted. There has been a 5-fold increase in incidence over the past decade, with increasing age being an important risk factor [2]. It can be caused by group A Streptococcal infection often incombination with Staphlococcus Aureus. It may also be polymicrobial with several aerobic and anaerobic organisms implicated, including Bacteroides, Clostridium, Peptostreptococcus, Enterobacteriaceae, coliforms, Proteus, Pseudomonas, and Klebsiella. Presentation can occur anywhere in the body but the lower limb is most commonly affected, varying from $32 \%-58 \%$ $[3,4]$. Diagnosis remains challenging and it is often missed in the early stages. Early fascial biopsy and histopathological analysis is useful in cases where the diagnosis is unclear. Magnetic Resonanace Imaging (MRI) may also be useful as it can differentiate between necrotising fasciitis and cellulites [5].

\section{Case Report}

A 82-year-old male who was referred a local district general hospital with a two week history of pain, redness, swelling and bruising of his left medial thigh; (Figure 1). He had failed to respond to standard intravenous antibi-

${ }^{*}$ Corresponding author. otic therapy for a presumed diagnosis of cellulitis. Upon presentation the thigh was very swollen and tense. There was no ulceration or other skin lesion noted.

Clinically he was in septic shock. His inflammatory markers were noted to be elevated (WCC 13.3, CRP 202), with a deteriorating renal function and markedly elevated lactate levels. Blood gas analysis showed a metaboloic acidosis. The patient was transferred to the intensive care unit (ICU) and commenced on intravenous Clindamycin, Ciprofloxacin and Vancomycin. Tissue for culture failed to isolate any organism.

MRI scan on the day of admission demonstrated skin and subcutaneous thickening with oedema of the posterior compartment crossing the fascial planes to the anterior compartment (Figure 1). The muscles of the medial compartment of the upper leg were preserved but grossly oedematous (Figure 2). These findings in conjunction with the history were highly suggestive of a severe inflammatory process.

After resuscitation the patient underwent urgent surgical debridement under general anaesthesia. The intraoperative findings revealed overlying necrotic skin and fascia in the medial compartment of the thigh. There was thrombosis of cutaneous vessels. The underlying muscles were necrotic and there was extensive, purulent appearing exudate. Samples were sent for histology and microbiology. A presumptive diagnosis of necrotising fasciitis was made.

The patient was returned to ICU where he continued to 


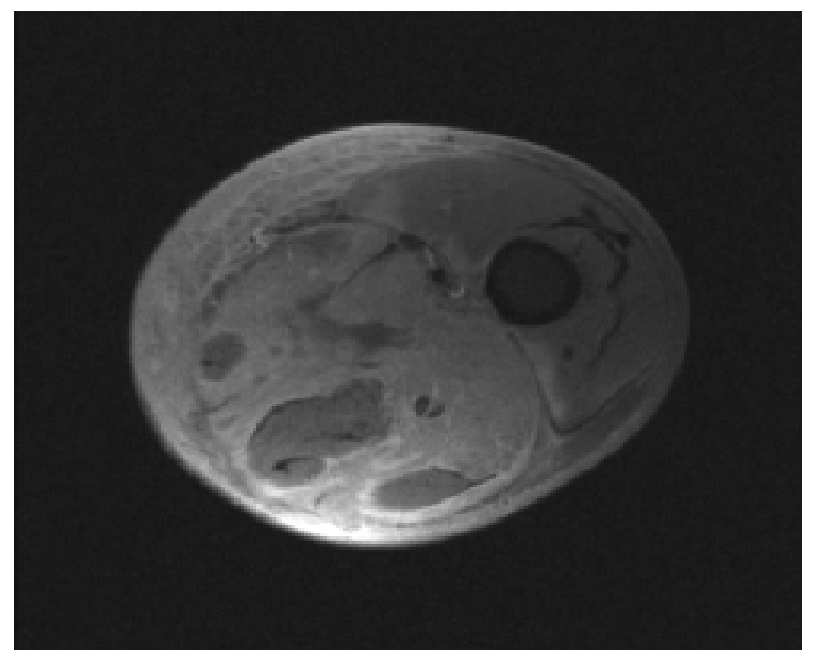

Figure 1. Axial MRI image of left thigh showing gross oedema of the posterior compartment crosssing the fascial planes into the anterior compartment.

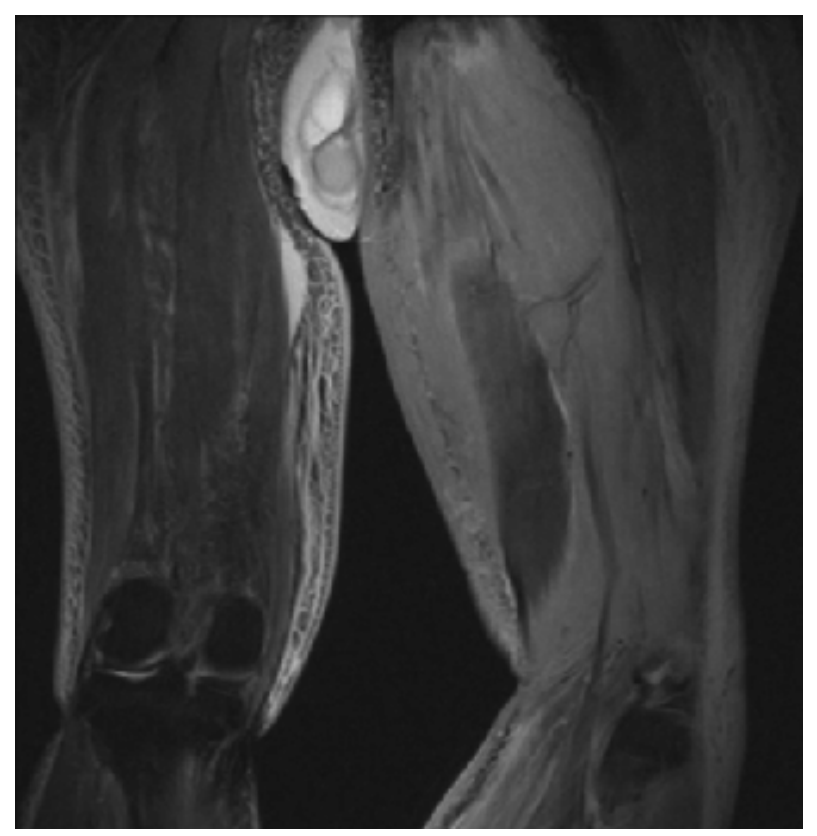

Figure 2. Saggital MRI image of the left thigh showing skin thickening of the left medial thigh with adductor oedema.

be administered with intravenous antibiotics. He also required haemodialysis and inotropic support. Further debridements were undertaken on the third and fifth day. A negative pressure dressing was applied. Although there was no clinical evidence of infection, haematological indices including CRP (325), WBC (21.4) and lactate dehydrogenase (22 (normal 0.05 - 2.20)) continued to deteriorate. A Computed Tomography (CT) scan of thorax, abdomen and pelvis showed no other occult sepsis. On day 15 the wounds were covered with split thickness skin graft (Figure 3).

A diagnosis of primary cutaneous large B-cell lym-

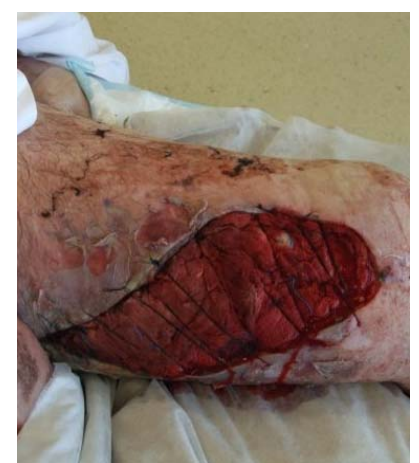

(a)

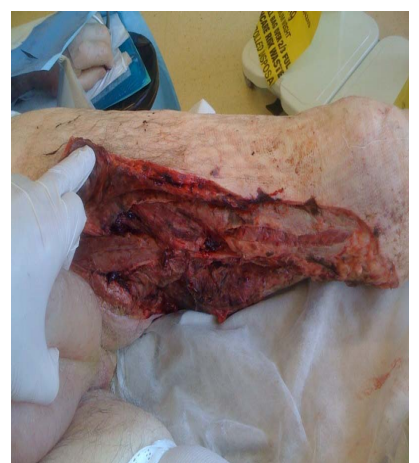

(b)

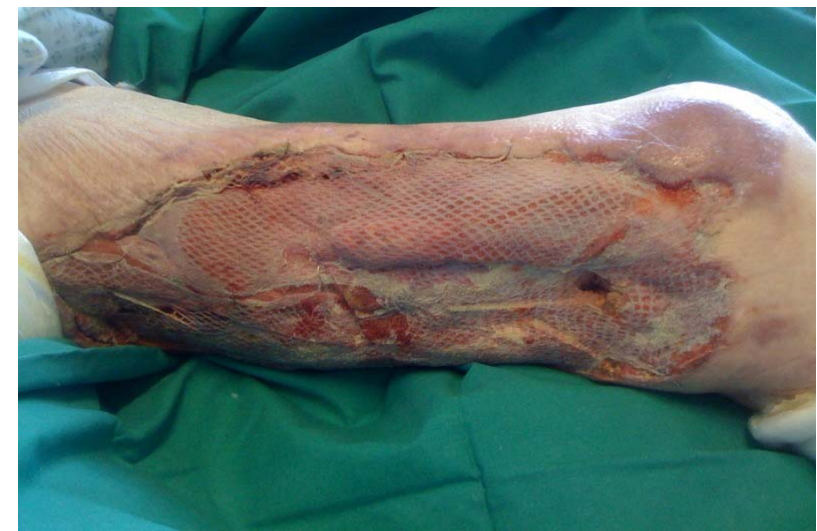

(c)

Figure 3. (a) Day 2 wound debridement: wound internally packed with bethadine soaked gauze. (b), (c) Day 14 wound debridement and split thickness skin grafting. Note a clean wound with healthy skin edges and significant amount of muscle resected. Note the extension of the lymphoma over the patella.

phoma of the leg (PCLBCL-leg) was made after histological analysis of the fascia and attached adipose tissue showed diffuse infiltration by atypical lymphoid cells (Figure 4). The proliferation of lymphoid cells shows so-called starry-sky appearance with the presence of large histiocytes phagocyting nuclear debris. Mitotic figures were also present. The atypical lymphoid cells showed CD20 and BCL6 positivity.

He was referred to the haematology service, but he continued to deteriorate in ITU and died before chemotherapy was commenced.

\section{Discussion}

Necrotising fasciitis typically presents with early signs of hot overlying skin, intense erythema, small serous bullae, and firm induration signifying underlying fascial involvement but may appear as mild cellulites [6]. Connective tissue and fat is broken down by hyaluronidase and lipases released by bacteria which allows rapid spread of infection along fascial planes. Aggressive debridement is the fundamental principle in the treatment of necrotising 


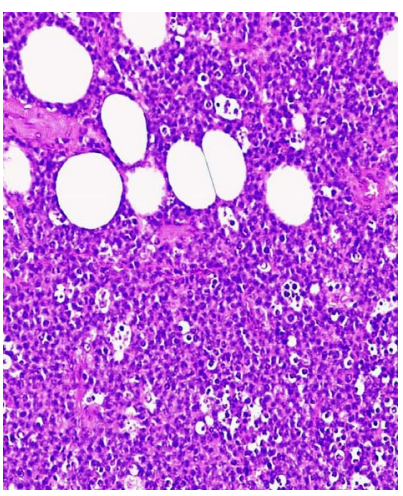

(a)

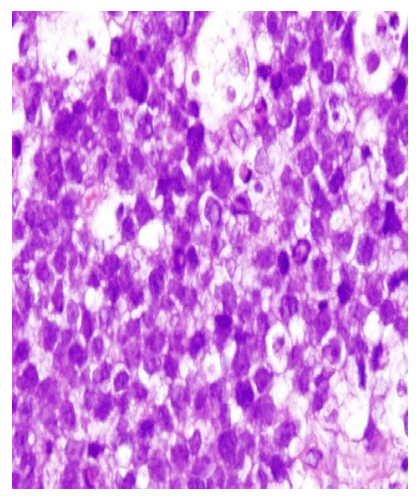

(b)

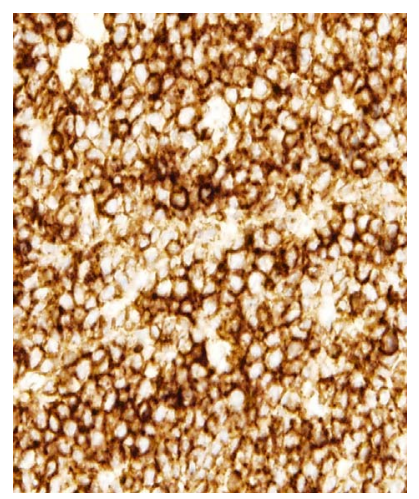

(c)

Figure 4. (a) Adipose tissue shows diffuse infiltraion by lymphoid cells (×10); (b) Atypical lymphoblasts and immunoblasts predominate the picture with several mitotic figures and apoptotic bodies (×40); (c) CD20 immunohistochemistry shows strong positivity confirming B-cell origin of the lesion.

fasciitis [7]. The value of obtaining a fresh frozen biopsy is evident not only in terms of improved diagnosis but also survival $[8,9]$.

Various B-cell lymphomas can present primarily in the skin without evidence of extracutaneous disease [10]. Primary cutaneous large B-cell lymphomas [PCLBCLs] are responsible for the majority of these [11]. These lypmhomas are divided into three main subtypes; primary cutaneous marginal zone B-cell lymphoma (PCMZL), primary cutaneous follicle center-cell lymphomas (PCFCCLs) and primary cutaneous large B-cell lymphoma of the leg (PCLBCL-leg). PCLBCL-leg differs by being rapidly progressive, having a poorer prognosis, higher age of onset, and expressing bcl-2 protein in the vast majority of cases $[10,12]$. The neoplastic $B$ cells also express the B-cell-associated antigen CD20. PCLBCL-leg develop extracutaneous disease more frequently and patients have a 5-year survival of 52\% compared with $94 \%$ with PCFCCL [13].

PCLPBL commonly presents with one or both legs exhibiting a rapidly growing red or bluish tumour (Is it cutaneous or subcutaneous?). An elevated serum lactate deydrogenase has been identified in $11.7 \%$ of cases [14]. Approximately 25\% of patients will develop extracutaneous disease at a mean time of 22 months while rarely lesions may present in a primary location other than the leg and carry a better prognosis $[13,15]$.

Radiotherapy, chemotherapy and immunotherapy are the mainstays of treatment [17]. Regimens typically include an anti-CD20 antibody (rituximab) in comination with the antracycline based chemotherapy regimen CHOP (r-CHOP) $[15,17,18]$. Radiotherapy may also be considered particularly in presentations of a solitary small skin tumour. Relapse rates following radiation have been reported as $58 \%$ with extracutaneous progression rates of $30 \%$ [17]. Surgical excision is highly effective in PCMZL and PCFCCL with a similar efficacy to radiotherapy, however its efficacy is not well described in PCLPBL [19].

This case highlights the need for urgent tissue diagnosis even when you think the diagnosis is obvious. Had an earlier tissue diagnosis been made Initial treatment of this patient with radiation and/or r-CHOP may have improved his prognosis. The presence of a raised lactate should also lead one to suspect cutaneous lymphoma. We therefore recommend that urgent tissue biopsy be performed in all cases of presumed necrotising fasciitis.

\section{REFERENCES}

[1] E. J. Whallett, J. H. Stevenson and A. D. Wilmshurst, "Necrotising Fasciitis of the Extremity," Journal of Plastic, Reconstructive \& Aesthetic Surgery, Vol. 63, No. 5, 2010, pp. e469-e473. doi:10.1016/j.bjps.2009.09.011

[2] R. Kaul, A. McGeer, D. E. Low, et al., "PopulationBased Surveillance for Group a Streptococcal Necrotizing Fasciitis: Clinical Features, Prognostic Indicators, and Microbiologic Analysis of Seventy-Seven Cases. Ontario Group A Streptococcal Study,” American Journal of Medicine, Vol. 103, No. 1, 1997, pp. 18-24. doi:10.1016/S0002-9343(97)00160-5

[3] D. A. Anaya and E. P. Dellinger, "Necrotizing Soft-Tissue Infection: Diagnosis and Management," Clinical Infectious Diseases, Vol. 44, No. 5, 2007, pp. 705-710. doi:10.1086/511638

[4] B. J. Childers, L. D. Potyondy, R. Nachreiner, et al., "Necrotizing Fasciitis: A Fourteen-Year Retrospective Study of 163 Consecutive Patients," The American Journal of Surgery, Vol. 68, No. 2, 2002, pp. 109-116.

[5] A. Rahmouni, O. Chosidow, D. Mathieu, et al., "MR Imaging in Acute Infectious Cellulites,” Radiology, Vol. 192, No. 2, 1994, pp. 493-496.

[6] J. D. Urschel, "Necrotizing Soft Tissue Infections," Postgraduate Medical Journal, Vol. 75, No. 889, 1999, pp. 645-649. doi:10.1136/pgmj.75.889.645

[7] C. H. Wong, A. K. Yam, A. B. Tan, et al., "Approach to Debridement in Necrotizing Fasciitis," The American Journal of Surgery, Vol. 196, No. 3, 2008, pp. e19-e24. 
doi:10.1016/j.amjsurg.2007.08.076

[8] I. Stamenkovic and P. D. Lew, "Early Recognition of Potentially Fatal Necrotizing Fasciitis. The Use of Frozen-Section Biopsy,” The New England Journal of Medicine, Vol. 310, No. 26, 1984, pp. 1689-1693. doi:10.1056/NEJM198406283102601

[9] J. Majeski and E. Majeski, "Necrotizing Fasciitis: Improved Survival with Early Recognition by Tissue Biopsy and Aggressive Surgical Treatment," Southern Medical Journal, Vol. 90, No. 11, 1997, pp. 1065-1068. doi:10.1097/00007611-199711000-00001

[10] R. Willemze, H. Kerl, W. Sterry, et al., "EORTC Classification for Primary Cutaneous Lymphomas: A Proposal from the Cutaneous Lymphoma Study Group of the European Organization for Research and Treatment of Cancer,” Blood, Vol. 90, No. 1, 1997, pp. 354-371.

[11] P. Isaacson and A. Norton, "Cutaneous Lymphomas," In: P. G. Isaacson and N. A. Wright, Eds., Extranodal Lymphomas, Churchill Livingstone, London, 1994, p. 172.

[12] F. A. Geelen, M. H. Vermeer, C. J. Meijer, et al., "Bcl-2 Protein Expression in Primary Cutaneous Large B-Cell Lymphoma is Site-Related," Journal of Clinical Oncology, Vol. 16, No. 6, 1998, pp. 2080-2085.

[13] F. Grange, M. W. Bekkenk, J. Wechsler, et al., "Prognostic Factors in Primary Cutaneous Large B-Cell Lymphomas: A European Multicenter Study,” Journal of Clinical Oncology, Vol. 19, No. 16, 2001, pp. 3602-3610.

[14] F. Grange, M. Beylot-Barry, P. Courville, et al., "Primary Cutaneous Diffuse Large B-Cell Lymphoma, Leg Type: Clinicopathologic Features and Prognostic Analysis in 60
Cases,” Archives of Dermatology, Vol. 143, No. 9, 2007, pp. 1144-1150. doi:10.1001/archderm.143.9.1144

[15] R. Willemze, E. S. Jaffe, G. Burg, et al., "WHO-EORTC Classification for Cutaneous Lymphomas,” Blood, Vol. 105, No. 10, 2005, pp. 3768-3785. doi:10.1182/blood-2004-09-3502

[16] P. L. Zinzani, P. Quaglino, N. Pimpinelli, et al., "Prognostic Factors in Primary Cutaneous B-Cell Lymphoma: the Italian Study Group for Cutaneous Lymphomas," Journal of Clinical Oncology, Vol. 24, No. 9, 2006, pp. 1376-1382. doi:10.1200/JCO.2005.03.6285

[17] N. J. Senff, E. M. Noordijk, Y. H. Kim, et al., "European Organization for Research and Treatment of Cancer and International Society for Cutaneous Lymphoma Consensus Recommendations for the Management of Cutaneous B-Cell Lymphomas,” Blood, Vol. 112, No. 5, 2008, pp. 1600-1609. doi:10.1182/blood-2008-04-152850

[18] B. Dreno, "Standard and New Treatments in Cutaneous B-Cell Lymphomas,” Journal of Cutaneous Pathology, Vol. 33, No. S1, 2006, pp. 47-51. doi:10.1111/j.0303-6987.2006.00544.x

[19] P. Golling, A. Cozzio, R. Dummer, et al., "Primary Cutaneous B-Cell Lymphomas-Clinicopathological, Prognostic and Therapeutic Characterisation of 54 Cases according to the WHO-EORTC Classification and the ISCL/EORTC TNM Classification System for Primary Cutaneous Lymphomas Other than Mycosis Fungoides and Sezary Syndrome," Leuk Lymphoma, Vol. 49, No. 6, 2008, pp. 1094-1103. doi:10.1080/10428190802064925 